\title{
Papers
}

\section{Changes in blood pressure among students attending Glasgow University between 1948 and 1968: analyses of cross sectional surveys}

\author{
Peter McCarron, Mona Okasha, James McEwen, George Davey Smith
}

\begin{abstract}
Objectives To examine the changes in blood pressure over time in a cohort of young adults attending university between 1948 and 1968 .

Design Cross sectional study.

Setting Glasgow University.

Participants 12414 students aged 16-25 years-9248

men (mean age 19.9 years) and 3164 women (19.2

years) - who participated in health screening on entering university between 1948 and 1968.

Main outcome measures Systolic and diastolic blood pressure.

Results In male students mean systolic blood pressure adjusted for age decreased from 134.5 (95\% confidence interval 133.8 to 135.2$) \mathrm{mm} \mathrm{Hg}$ in those born before 1929 to 125.7 (125.0 to 126.3 ) $\mathrm{mm} \mathrm{Hg}$ in those born after 1945, and diastolic blood pressure dropped from 80.3 (79.8 to 80.8$) \mathrm{mm} \mathrm{Hg}$ to 74.7 (74.2 to 75.1$) \mathrm{mm} \mathrm{Hg}$. For female students the corresponding declines were from 129.0 (127.5 to 130.5$) \mathrm{mm} \mathrm{Hg}$ to 120.6 (119.8 to 121.4) $\mathrm{mm} \mathrm{Hg}$ and from 79.7 (78.7 to 80.6) $\mathrm{mm} \mathrm{Hg}$ to 77.0 (76.5 to 77.5) $\mathrm{mm} \mathrm{Hg}$. Adjustment for potential confounding factors made little difference to these findings. The proportion of students with hypertension declined substantially in both sexes. Conclusions Substantial declines in systolic and diastolic blood pressure over time were occurring up to 50 years ago in young adults who were not taking antihypertensive medication. Since blood pressure tracks into adult life, the results of the cross sectional comparisons suggest that factors acting in early life may be important in determining population risk of cardiovascular disease. Changes in such factors may have made important contributions to the decline in rates of cardiovascular diseases, particularly stroke, seen in developed countries during the past century.
\end{abstract}

\section{Introduction}

Mortality from stroke has declined dramatically in developed countries throughout most of the past century, ${ }^{12}$ and more recently mortality from coronary heart disease has also decreased. ${ }^{1}$ Raised blood pressure is a major modifiable risk factor for both conditions. ${ }^{3}$ Since blood pressure in young adults is also positively associated with mortality from cardiovascular disease in later life, ${ }^{4}$ decreases in blood pressure in this age group could underlie declines in mortality from cardiovascular disease.

Blood pressure tracks from childhood through to later life. ${ }^{5}$ Examining trends in blood pressure in young adults, in whom blood pressure is largely uninfluenced by disease, medication, or behavioural changes consequent on morbidity, can help to elucidate the population determinants of blood pressure. To date, however, few studies have investigated such trends. ${ }^{6} \mathrm{We}$ report on the changes in blood pressure over time in a cohort of Glasgow University students born between 1925 and 1950.

\section{Methods}

Full details of the study are available elsewhere. ${ }^{7}$ Briefly, students attending Glasgow University between 1948 and 1968 were invited to participate in an ongoing health survey consisting of a questionnaire and clinical examination. Data collected included sociodemographic details, health behaviours, and medical history. Height, weight, and blood pressure were also recorded.

A total of 15322 students (11 755 men and 3567 women) participated in the study. Students born before 1925 (319), with unknown date of birth (2), or aged over 25 years at examination (1324) were excluded from the analyses, as were two students with lower systolic than diastolic blood pressure and 104 students without a record of blood pressure. Students with missing data on potential confounding variables (1159) were also excluded.

\section{Statistical analyses}

Year of birth was divided into five bands: 1925-9, 1930-4, 1935-9, 1940-4, and 1945-50. Linear regression analyses were used to examine trends in blood pressure. Fully adjusted mean blood pressures were then calculated, controlled for the effects of smoking (yes/no), height (metres), body mass index $\left(\mathrm{kg} / \mathrm{m}^{2}\right)$, father's social class $(\mathrm{I}-\mathrm{V})$, and age at menarche $(\leqslant 11,12,13,14, \geqslant 15$ years). Likelihood ratio tests were used to test for a linear trend in blood pressure for each one year increase in birth year. To assess the change over time in the proportion of individuals with

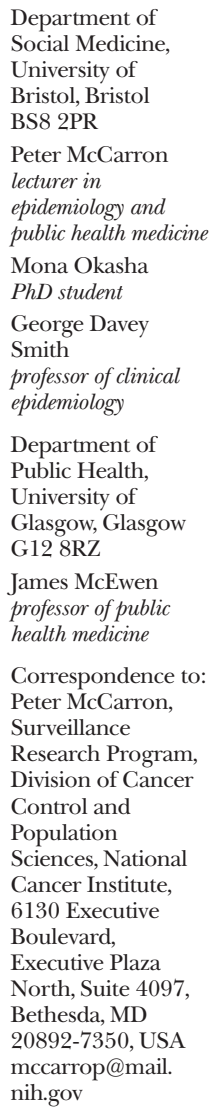

Department of Social Medicine, University of Bristol, Bristol BS8 2PR

Peter McCarron lecturer in epidemiology and public health medicine Mona Okasha PhD student

George Davey Smith professor of clinical epidemiology

Department of Public Health, University of Glasgow, Glasgow G12 8RZ

James McEwen professor of public health medicine

Correspondence to: Peter McCarron, Surveillance

Research Program, Division of Cancer

Control and Population Sciences, National Cancer Institute, 6130 Executive Boulevard, Executive Plaza North, Suite 4097, Bethesda, MD 20892-7350, USA mccarrop@mail. nih.gov

BMJ 2001;322:885-9 
Table 1 Blood pressure $(\mathrm{mm} \mathrm{Hg})$ in male and female students by birth band. Values are means ( $95 \%$ confidence intervals) unless stated otherwise

\begin{tabular}{|c|c|c|c|c|}
\hline \multirow[b]{2}{*}{ Date of birth } & \multicolumn{2}{|c|}{ Adjusted for age } & \multicolumn{2}{|c|}{ Fully adjusted ${ }^{*}$} \\
\hline & Systolic blood pressure & Diastolic blood pressure & Systolic blood pressure & Diastolic blood pressure \\
\hline \multicolumn{5}{|l|}{ Male students $(n=9248)$ : } \\
\hline $1925-9$ & $134.2(133.5$ to 134.9$)$ & 80.2 (79.7 to 80.6$)$ & 134.5 (133.8 to 135.2$)$ & 80.3 (79.8 to 80.8$)$ \\
\hline $1930-4$ & 132.6 (132.1 to 133.2) & 78.4 (78.1 to 78.8$)$ & 132.6 (132.1 to 133.2) & 78.4 (78.1 to 78.8$)$ \\
\hline $1935-9$ & 131.4 (130.8 to 131.9) & 76.2 (75.9 to 76.6$)$ & 131.4 (130.8 to 131.9) & 76.3 (75.9 to 76.6$)$ \\
\hline $1940-4$ & 130.2 (129.6 to 130.8$)$ & 75.5 (75.1 to 75.9$)$ & 130.0 (129.5 to 130.7$)$ & 75.5 (75.1 to 75.9$)$ \\
\hline $1945-50$ & 125.9 (125.2 to 126.5$)$ & 74.8 (74.4 to 75.2$)$ & 125.7 (125.0 to 126.3 ) & 74.7 (74.2 to 75.1$)$ \\
\hline$P$ for trend for 1 year increase & $<0.001$ & $<0.001$ & $<0.001$ & $<0.001$ \\
\hline \multicolumn{5}{|l|}{ Female students $(\mathrm{n}=3164)$ : } \\
\hline $1925-9$ & $129.0(127.7$ to 130.4$)$ & 79.6 (78.7 to 80.6$)$ & 129.0 (127.5 to 130.5$)$ & 79.7 (78.7 to 80.6$)$ \\
\hline $1930-4$ & 128.9 (128.1 to 129.6) & 79.7 (79.2 to 80.2$)$ & 128.7 (127.9 to 129.5$)$ & 79.6 (79.1 to 80.1$)$ \\
\hline $1935-9$ & $123.0(122.0$ to 123.9$)$ & 76.5 (75.9 to 77.1$)$ & 122.9 (121.9 to 123.8$)$ & 76.4 (75.8 to 77.0$)$ \\
\hline $1940-4$ & 120.8 (119.9 to 121.7) & 76.4 (75.8 to 77.0$)$ & 120.9 (120.1 to 121.8) & 76.5 (75.9 to 77.0$)$ \\
\hline $1945-50$ & 120.5 (119.7 to 121.3$)$ & 76.9 (76.4 to 77.4$)$ & 120.6 (119.8 to 121.4) & 77.0 (76.5 to 77.5$)$ \\
\hline$P$ for trend for 1 year increase & $<0.001$ & $<0.001$ & $<0.001$ & $<0.001$ \\
\hline
\end{tabular}

${ }^{*}$ Controlling for age, height, body mass index, smoking, father's social class, and age at menarche in women.

normal and high blood pressure, blood pressure adjusted for age was categorised as optimal $(<120 / 80$ $\mathrm{mm} \mathrm{Hg})$, normal (120-129/80-84 mm Hg), high normal (130-139/85-89 mm Hg, hypertension stage 1 (140-159/90-99 mm $\mathrm{Hg})$, hypertension stage 2 (160-179/100-109 mm Hg), or hypertension stage 3 $(\geqslant 180 / 110 \mathrm{~mm} \mathrm{Hg})^{8}$ and presented for different birth bands. Trend in blood pressure category was examined by using ordinal logistic regression. Analyses were carried out with Stata 6.0. ${ }^{9}$

Table 2 Blood pressure category in male and female students. ${ }^{*}$ Values are numbers (percentages)

\begin{tabular}{lccc} 
Blood pressure group & $\begin{array}{c}\text { Male students } \\
(\mathbf{n = 9 2 4 8 )}\end{array}$ & $\begin{array}{c}\text { Female students } \\
(\mathbf{n}=\mathbf{3 1 6 4 )}\end{array}$ & $\begin{array}{c}\text { Total } \\
(\mathbf{n = 1 2 4 1 2})\end{array}$ \\
\hline Optimal & $961(10.4)$ & $768(24.3)$ & $1729(13.9)$ \\
\hline Normal & $2566(27.8)$ & $1171(37.0)$ & $3737(30.1)$ \\
\hline High normal & $2763(29.9)$ & $691(21.8)$ & $3454(27.8)$ \\
\hline Hypertension (stage 1) & $2584(27.9)$ & $493(15.6)$ & $3007(24.8)$ \\
\hline Hypertension (stage 2) & $330(3.6)$ & $40(1.3)$ & $370(3.0)$ \\
\hline Hypertension (stage 3) & $44(0.5)$ & $1(0)$ & $45(0.4)$ \\
\hline
\end{tabular}

Table 3 Prevalence of blood pressure (adjusted for age) categories by birth band

\begin{tabular}{|c|c|c|c|c|c|c|}
\hline \multirow[b]{2}{*}{ Blood pressure group } & \multicolumn{6}{|c|}{ Prevalence $(\%)$ in each five year birth band } \\
\hline & $1925-9$ & $1930-4$ & $1935-9$ & $1940-4$ & $1945-50$ & Total \\
\hline \multicolumn{7}{|l|}{ Male students: } \\
\hline Optimal & 17.4 & 12.3 & 14.7 & 20.7 & 21.6 & 17.0 \\
\hline Normal & 30.3 & 25.0 & 27.3 & 28.2 & 35.2 & 28.9 \\
\hline High normal & 31.5 & 31.6 & 29.7 & 25.5 & 24.4 & 28.7 \\
\hline Hypertension (stage 1) & 18.0 & 27.8 & 25.0 & 21.6 & 16.5 & 22.3 \\
\hline Hypertension (stage 2) & 2.4 & 2.9 & 3.1 & 3.5 & 2.1 & 2.8 \\
\hline Hypertension (stage 3) & 0.4 & 0.3 & 0.1 & 0.4 & 0.2 & 0.3 \\
\hline All & 100 & 100 & 100 & 100 & 100 & 100 \\
\hline & \multicolumn{6}{|c|}{ Test for trend: $\mathrm{P}<0.001$} \\
\hline \multicolumn{7}{|l|}{ Female students: } \\
\hline Optimal & 17.0 & 22.7 & 35.8 & 38.9 & 38.6 & 32.0 \\
\hline Normal & 33.9 & 30.4 & 35.0 & 37.2 & 38.6 & 35.1 \\
\hline High normal & 28.7 & 23.8 & 20.4 & 16.0 & 15.6 & 19.9 \\
\hline Hypertension (stage 1) & 19.4 & 21.0 & 8.3 & 7.4 & 7.1 & 12.1 \\
\hline Hypertension (stage 2) & 1.0 & 2.1 & 0.5 & 0.5 & 0.2 & 0.9 \\
\hline Hypertension (stage 3) & 0 & 0 & 0 & 0 & 0 & 0 \\
\hline All & 100 & 100 & 100 & 100 & 100 & 100 \\
\hline
\end{tabular}

\section{Results}

After exclusions, 12412 students (9248 men and 3164 women) remained eligible for analysis. Male students were slightly older than female ones, with a mean age of 19.9 (SD 1.8) years versus 19.2 (1.4) years. Systolic blood pressure was higher in male students (130.9 (13.0) $\mathrm{mm} \mathrm{Hg}$ ) than in female students (124.0 (12.1) $\mathrm{mm} \mathrm{Hg}$ ), whereas diastolic blood pressure was marginally lower in male students (77.0 (8.6) $\mathrm{mm} \mathrm{Hg} v 77.7$ (7.5) $\mathrm{mm} \mathrm{Hg}$ ).

Trends in blood pressure

Table 1 shows the changes in blood pressure with increasing birth band. As results adjusted for age were similar for the full cohort and for those people with data on confounding variables, only the latter are reported here. There was little difference between blood pressure adjusted for age and fully adjusted blood pressure.

When year of birth was entered as a continuous variable, systolic and diastolic blood pressure in men declined by 0.40 (95\% confidence interval 0.36 to 0.45$)$ $\mathrm{mm} \mathrm{Hg}$ and 0.28 ( 0.25 to 0.30$) \mathrm{mm} \mathrm{Hg}$ respectively per one year increase in year of birth. For women the corresponding declines were 0.55 (0.48 to 0.61$) \mathrm{mm} \mathrm{Hg}$ and $0.19(0.15$ to 0.23$) \mathrm{mm} \mathrm{Hg}$. Controlling for confounding variables had little effect on these findings.

\section{Trends in hypertension}

Most students had optimal, normal, or high normal blood pressure (table 2). Most students with high blood pressure had stage 1 hypertension; $87.4 \%$ of all men with hypertension and $92.3 \%$ of all women with hypertension were in this category. Table 3 shows a linear increase in normotension and decrease in hypertension with increasing birth band in both sexes $(\mathrm{P}<0.0001)$.

\section{Discussion}

Substantial downward trends in blood pressure occurred in male and female students attending the University of Glasgow between 1948 and 1968, and remained after confounding factors were controlled for. 


\section{Strengths and weaknesses of the study}

The current study is large, and data are available for both sexes. As details of how blood pressure was recorded and of the identity of the observers were not available we cannot assess observer variation. Almost $74 \%$ of valid readings ended in 0 or 5 ; however, although this might attenuate the magnitude of any trend uncovered, it would have little effect on the direction of these associations. ${ }^{10}$ Also, as diastolic blood pressure in women was lower than that in men at the beginning of the study period but higher at the end, and as any systematic change in measurement protocol should have affected readings in both sexes similarly, it is unlikely that such a change could have resulted in the substantial trends observed.

\section{Trends in blood pressure}

In a study from Queen's University, Belfast, blood pressure declined during the period 1948-70 from $131 / 78 \mathrm{~mm} \mathrm{Hg}$ to $123 / 72 \mathrm{~mm} \mathrm{Hg}$ in male students and from $122 / 78 \mathrm{~mm} \mathrm{Hg}$ to $110 / 70 \mathrm{~mm} \mathrm{Hg}$ in female students. ${ }^{11}{ }^{12}$ US national health and nutrition examination surveys from 1960 to 1991 showed small increments in both mean systolic and mean diastolic blood pressure in men and women aged 18-29 years between the first two surveys (1960-2 and 1971-4), but since then blood pressure has declined in both sexes. ${ }^{6}$ A limitation of the above studies is that the role of confounding was not adequately assessed.

\section{Trends in hypertension}

The prevalence of hypertension in the current study is similar to that in the Johns Hopkins precursors study. ${ }^{13}$ In that study, which was smaller and restricted to male medical students, 3.5\% of participants (mean age 22 years) who graduated between 1948 and 1964 had grade 2 hypertension or worse compared with $2.5 \%$ of male students in Glasgow. In the US national health and nutrition examination surveys the prevalence of stage 2 hypertension or worse in white men aged 18-29 increased between the first and third surveys and then declined, whereas in similarly aged women the prevalence showed a continual decline over the survey period, 1960-91. ${ }^{6}$

\section{Explanation of trends}

The substantial decline in blood pressure is not accounted for by confounding factors. Neither can the use of antihypertensive drugs explain the trends reported here as most of the measurements were carried out before antihypertensive drugs were available and to our knowledge none of the students was taking such medication.

A recent systematic review of trials of salt restriction reported that blood pressure was lower when salt intake was reduced, although the effects were small. ${ }^{14}$ However, in adolescents randomised in infancy to a low or a normal sodium diet, blood pressure was lower after 15 years in individuals in the intervention arm compared with those in the control group, suggesting that sodium restriction in infancy may have greater effects on later blood pressure than reduction in salt intake in adulthood. ${ }^{15}$ Although caution is required in interpreting these findings as the proportion of participants followed up was low, alterations in salt intake in early life, through dietary change and possibly through increasing use of refrigeration to

\section{What is already known on this topic}

Mortality from stroke in developed countries decreased throughout the 20th century; declines in mortality from coronary heart disease occurred more recently

Explanations may include favourable trends in risk factors, including blood pressure

Since blood pressure tracks into adulthood, description of the trends occurring in blood pressure in young adults may help to elucidate the critical periods involved in influencing trends in blood pressure

\section{What this study adds}

Declines in blood pressure over time were taking place up to 50 years ago in young adults

These changes did not result from use of antihypertensive medications and point to the importance of factors in early life in determining blood pressure and risk of subsequent cardiovascular disease

store foodstuffs, ${ }^{1}$ may explain some of the decline in blood pressure found in the current study.

Other dietary changes during the period when most of participants in this study were born and grew up may be important. Between 1903 and 1934 there were large increases in fruit and vegetable consumption, especially among affluent people - the group from which most of the participants in this study came. ${ }^{16}$ Changes in consumption of other foods were proportionately much less. Accurate assessment of levels of dietary constituents in the first half of the last century is problematic, but there was certainly a trend towards increasing consumption of vegetables, fruit, and cereals. Importantly, fruit and vegetables, unlike other foods, were not rationed during the second world war, resulting in a general improvement in quality of diet across the social spectrum. ${ }^{17-19}$ That such changes may have contributed to a progressively more favourable blood pressure profile is supported by an American dietary trial in which there were substantial declines in blood pressure in people assigned diets rich in fruit and vegetables compared with controls on a more typical American diet. ${ }^{20}$

Most studies have reported that birth weight is inversely associated with subsequent blood pressure. ${ }^{21}$ However, even if the increase in birth weight in the United Kingdom since at least the 1970s had been occurring throughout the last century the increment is likely to have been modest, and certainly no more than $550 \mathrm{~g}$ in Scotland over the century (Information and Statistics Division, Common Services Agency, Scotland: unpublished SMR2 data). ${ }^{22}{ }^{23}$ Since it has been estimated that a $1000 \mathrm{~g}$ rise in birth weight is required for a $2 \mathrm{~mm}$ $\mathrm{Hg}$ drop in systolic blood pressure in people at age $50,{ }^{21}$ any upward trend in birth weight in the current cohort could not explain the large declines in blood pressure seen. Finally, while it seems that postnatal growth has a role in determining later blood pressure this is poorly understood and findings are conflicting. ${ }^{24-26}$ 


\section{Conclusion}

The substantial declines in blood pressure occurring in young adults between 30 and 50 years ago in the United Kingdom may have had their origin in early life and may explain some of the decline in rates of cardiovascular disease over the past century.

We thank Alan Kerr, Christine Hamilton, and Heather Learmonth for entering the data and Jonathon Sterne for advice on statistical analyses.

Contributors: PMcC, GDS, and JMcE discovered the original data and designed the study. PMcC carried out the analyses and drafted the paper. GDS, MO, and JMcE commented critically on subsequent drafts. PMcC will act as guarantor.

Funding: Chest, Heart and Stroke, Scotland; Stroke Association; NHS Management Executive; Cardiovascular Disease and Stroke Research and Development Initiative.

Competing interests: None declared.

1 Charlton J, Murphy M. The health of adult Britain 1841-1994. London: Stationery Office, 1997

2 Bonita R, Stewart A, Beaglehole R. International trends in stroke mortality: 1970-1985. Stroke 1990;21:989-92.

3 Medical Research Council Working Party. MRC trial of treatment of mild hypertension: principal results. BMJ 1985;291:97-104.

4 McCarron P, Davey Smith G, Okasha M, McEwen J. Blood pressure in young adulthood and mortality from cardiovascular disease. Lancet 2000;355:1430-1.

5 Nelson MJ, Ragland DR, Syme SL. Longitudinal prediction of adult blood pressure from juvenile blood pressure levels. Am J Epidemiol 1992;136:633-45

6 Burt VL, Cutler JA, Higgins M, Horan MJ, Labarthe D, Whelton P, et al. Trends in the prevalence, awareness, treatment, and control of hypertension in the adult US population: data from the health examination surveys, 1960 to 1991. Hypertension 1995;26:60-9.

7 McCarron P, Davey Smith G, Okasha M, McEwen J. Life course exposure and later disease: a follow-up study based on medical examinations carried out in Glasgow University (1948-68). Public Health 1999;113:265-71.

8 Joint National Committee on Prevention, Detection, Evaluation, and Treatment of High Blood Pressure. The sixth report of the Joint National Committee of Prevention, Detection, Evaluation, and Treatment of High Blood Pressure. Arch Intern Med 1997;157:2413-46.
9 StataCorp. Stats Statistical Software: release 6.0. College Station, TX: Stata Corporation, 1998

10 Bennett S. Blood pressure measurement error: its effect on crosssectional and trend analyses. J Clin Epidemiol 1994;47:293-301.

11 Harland RW. Sociological, anatomical and physiological changes in firstyear students entering Queen's University, Belfast, over thirty years, 1948-77. Ulster Med J 1980;49:37-47.

12 Johnston W, Merrett JD. Further observations on routine medical examinations of university entrants in Northern Ireland. Br J Prev Soc Med 1962;16:76-83.

13 Thomas CB. Developmental patterns in hypertensive cardiovascular disease: fact or fiction? Bull NY Acad Med 1969;45:831.

14 Ebrahim S, Davey Smith G. Lowering blood pressure: a systematic review of sustained effects of non-pharmacological interventions. J Public Health Med 1998;20:441-8.

15 Geleijnse JM, Hofman A, Witteman JC, Hazebroek AA, Valkenburg HA, Grobbee DE. Long-term effects of neonatal sodium restriction on blood pressure. Hypertension 1997;29:913-7.

16 Orr JB. Food, health and income. London: Macmillan, 1936.

17 Ministry of Food. The urban working-class household diet 1940-1949. First report of the National Food Survey Committee. London: HMSO, 1951.

18 Baines AHJ, Hollingsworth DF, Leitch I. Diets of working-class families with children before and after the second world war. With a section on height and weight of children. Nutrition Abstracts and Reviews 1963;33:653-69.

19 Ministry of Food. Domestic food consumption and expenditure, 1950 (with a supplement on food expenditure by urban working-class households, 1940-1949). London: HMSO, 1952.

20 Appel LJ, Moore TJ, Obarzanek E, Vollmer WM, Svetkey LP, Sacks FM, et al. A clinical trial of the effects of dietary patterns on blood pressure. New Engl J Med 1997;336:1117-24.

21 Law CM, Shiell AW. Is blood pressure inversely related to birth weight? The strength of evidence from a systematic review of the literature. $J$ Hypertension 1996;14:935-41.

22 Power C. National trends in birth weight: implications for future adult disease. BMJ 1994;308:1270-1.

23 Ward WP. Birth weight and economic growth. Chicago and London: University of Chicago Press, 1993.

24 Berkey CS, Gardner J, Colditz GA. Blood pressure in adolescence and early adulthood related to obesity and birth size. Obes Res 1998;6:187-95.

25 Falkner B, Hulman S, Kushner H. Birth weight versus childhood growth as determinants of adult blood pressure. Hypertension 1998;31:145-50.

26 Montgomery SM, Berney LR, Blane D. Prepubertal stature and blood pressure in early old age. Arch Dis Child 2000;82:358-63.

(Accepted 23 January 2001)

\section{Commentary: Changing population blood pressure levels}

Bruce Neal

Institute for International Health, University of Sydney, PO Box 576, Newtown, Sydney, NSW 2042, Australia Bruce Neal programme director, heart and vascular diseases

bneal@med.usyd. edu.au
The level of blood pressure is a well established determinant of the risks of premature cardiovascular disease, ${ }^{1}$ and treatments to lower blood pressure have been shown to reduce these risks. ${ }^{2}$ Over the past 50 years efforts to prevent diseases related to blood pressure have concentrated primarily on drug based interventions among people with hypertension or a history of cardiovascular disease. However, blood pressure seems to be an important determinant of the risk of cardiovascular disease across the entire population, ${ }^{1}$ and it seems that even small population-wide reductions in blood pressure could prevent as many cardiovascular events as targeted clinical approaches. ${ }^{3}$ Unfortunately, effective methods for bringing about population-wide reductions in blood pressure are neither well established nor widely implemented.

McCarron et al provide strong evidence of decreases in mean blood pressure levels over time among students at Glasgow University between 1948 and 1968. The observed reductions are substantial and seem to be independent of any specific effort to modify blood pressure levels. The reasons for the observed changes in the blood pressure levels remain somewhat uncertain but seem likely to be a consequence of population-wide changes in the intake of dietary deter- minants of blood pressure, such as salt, fruit, and vegetables. Irrespective of the explanation, such a fall in blood pressure would be expected to produce a substantial reduction in the rate of premature cardiovascular disease. Data from a number of populations worldwide provide evidence of qualitatively similar changes in blood pressure that are likely to explain a non-trivial part of the reduction in mortality from stroke and other cardiovascular diseases reported by several countries over the past few decades.

If the reasons for the observed falls in blood pressure could be reliably determined this would facilitate the development of new strategies for the prevention of diseases related to blood pressure. Effective new preventive strategies aimed at broad population groups would be important in the United Kingdom as a whole but might be most relevant to economically disadvantaged people, for whom changes in cardiovascular mortality have not been favourable. ${ }^{5}$ However, possibly the greatest importance of such information would be for lower income countries in other parts of the world. These countries have the greatest burden of diseases related to blood pressure and over the next few decades will experience the greatest increases in such diseases. ${ }^{6}$ 
The work of McCarron et al has provided clear evidence that substantial changes in blood pressure levels can occur, and both these and other data suggest that such changes can occur in broad population groups. New epidemiological studies that provide reliable information about the most likely causes of these changes, and new large scale randomised trials that identify effective means of changing population levels of blood pressure, are needed. To have the greatest impact on the global burden of cardiovascular disease, however, the data from such studies must be directly applicable to the social, cultural, and economic circumstances of the less affluent sections of the global community.
Competing interests: None declared.

1 MacMahon S, Peto R, Cutler J, Collins R, Sorlie P, Neaton J, et al. Blood pressure, stroke, and coronary heart disease. Part 1. Prolonged differences in blood pressure: prospective observational studies corrected for the regression dilution bias. Lancet 1990;335:765-74

2 Blood Pressure Lowering Treatment Trialists' Collaboration. Effects of ACE inhibitors, calcium antagonists and other blood pressure lowering drugs: results of prospectively designed overviews of randomised trials. Lancet 2000;356:1955-64.

3 Rodgers A, Lawes C, MacMahon S. Reducing the global burden of blood pressure-related disease. J Hypertens 2000;18(suppl 1):S3-6.

4 Bonita R, Stewart A, Beaglehole R. International trends in stroke mortality: 1970-1985. Stroke 1990;21:989-92.

5 Marmot M, Adelstein A, Robinson N, Rose G. Changing social-class distribution of heart disease. BMJ 1978;2:1109-12.

6 Murray CJL, Lopez AD. Global patterns of cause of death and burden of disease in 1990, with projections to 2020. In: Investing in health research and development. Report of the Ad Hoc Committee on Health Research Relating to Future Intervention Options. Geneva: World Health Organization, 1996.

\title{
Birth weight and childhood onset type 1 diabetes: population based cohort study
}

\author{
Lars C Stene, Per Magnus, Rolv T Lie, Oddmund Søvik, Geir Joner and The Norwegian Childhood \\ Diabetes Study Group
}

\begin{abstract}
Objective To assess the associations between birth weight or gestational age and risk of type 1 diabetes. Design Population based cohort study by record linkage of the medical birth registry and the National Childhood Diabetes Registry.

Setting Two national registries in Norway. Participants All live births in Norway between 1974 and 1998 (1 382602 individuals) contributed a maximum of 15 years of observation, a total of 8184994 person years of observation in the period 1989 to 1998 . 1824 children with type 1 diabetes were diagnosed between 1989 and 1998 .

Main outcome measures Estimates of rate ratios with $95 \%$ confidence intervals for type 1 diabetes from Poisson regression analyses.

Results The incidence rate of type 1 diabetes increased almost linearly with birth weight. The rate ratio for children with birth weights $4500 \mathrm{~g}$ or more compared with those with birth weights less than $2000 \mathrm{~g}$ was 2.21 (95\% confidence interval 1.24 to 3.94), test for trend $\mathrm{P}=0.0001$. There was no significant association between gestational age and type 1 diabetes. The results persisted after adjustment for maternal diabetes and other potential

confounders.

Conclusion There is a relatively weak but significant association between birth weight and increased risk of type 1 diabetes consistent over a wide range of birth weights.
\end{abstract}

\section{Introduction}

Type 1 diabetes mellitus results from an immune mediated destruction of the pancreatic $\beta$ cells. The factors initiating the destructive process are largely unknown, but genetic and non-genetic factors are involved. ${ }^{1}$ Putative environmental risk factors such as viruses or nutritional factors may play a part early in life, possibly in utero. ${ }^{2}$ An association between high birth weight or high birth weight for gestational age and increased risk of type 1 diabetes has been found in some relatively large case-control studies, even after exclusion of data from children whose mother had diabetes in pregnancy. ${ }^{3-5}$ On the other hand, several other case-control studies have not found any significant association. ${ }^{6-14}$ The magnitude of the association between birth weight and type 1 diabetes seems to be relatively small, and the lack of significant association in the latter studies may be explained by insufficient statistical power. We estimated the associations between birth weight and gestational age and the incidence rate of type 1 diabetes in a large population based cohort study that provided sufficient power to estimate these associations over a wide range of values.

\section{Participants and methods}

\section{Participants}

Since the beginning of 1989 all newly diagnosed cases of type 1 diabetes diagnosed in children aged up to 15 years in Norway have been prospectively registered with a high level of ascertainment in the National Childhood Diabetes Registry. ${ }^{15}$ We designed a cohort study by record linkage of the Medical Birth Registry of Norway and the childhood diabetes registry through the unique personal identification number assigned to all residents of Norway. Out of 1863 cases of type 1 diabetes diagnosed between 1 January 1989 and 31 December 1998, 1824 were linked. All live births in Norway between 1974 and 1998 contributed time under observation from birth to diagnosis of type 1 diabetes, age 15 years, or 31 December 1998, whichever occurred first. As registration of cases started in 1989 , the time under observation was counted only from 1 January 1989 for those born before this date. Deaths in the first year of life were
Section of
Epidemiology,
Department of
Population Health
Sciences, National
Institute of Public
Health, PO Box
4404 Nydalen, 